\title{
Reliability based design optimization of semi-rigid steel frames
}

\author{
M. A. Hadianfard \\ Department of Civil Engineering, Shiraz University of Technology, Iran
}

\begin{abstract}
In the conventional methods of structural optimization, the designers reduce the structural cost or weight without taking into account uncertainties in the materials, geometry and loading. In this way, the optimum solution may represent a lower level of reliability and thus a higher risk of failure. However, the objective of reliability based design optimization (RBDO) is to design structures that are economic and reliable. In this paper, external loads and member resistance are assumed to be independent random variables. To identify critical failure modes, the $\beta$-unzipping method is used and the probability of failure of each mode is calculated using the method of reliability analysis of parallel systems. By combination of the different failure modes as a series system, the probability of failure of the total structural system is estimated. The effects of the semi-rigid behavior of the beam-column connections are also considered in the RBDO problem. The RBDO problem is formulated by the expected costs or structural weight as the objective function and the reliability of the system as constraints. This constrained nonlinear optimization problem is changed in to an unconstrained optimization problem using the method of interior penalty function. Then, this new problem is solved by an unconstrained optimization technique. Some numerical examples are presented to demonstrate the applicability of the proposed methodology. These examples illustrate the importance of the effect of the semi-rigid behavior of the connections in the calculation of the minimum weight of the steel frames.
\end{abstract}

Keywords: reliability, structural optimization, steel frames, semi-rigid connections, probability of failure. 


\section{Introduction}

The optimization of structures with respect to weight or cost is a well-known problem and it is the subject of many books, such as Kirsch [1] and Haftka and Gurdal [2]. However, the optimization of structures under reliability constraints is a complicated problem and it has been the subject of a few studies, such as Nakib and Frangopol [3]. The purpose of the reliability based design optimization (RBDO) is to produce reliable, and at the same token economical, structures. In the present study, by using a typical moment-rotation diagram, such as that described by Kishi et al. [4] and Hadianfard and Razani [5], the more realistic semi-rigid behavior of beam-column connections is considered in the structural analysis and in the RBDO. External loading, the resistance capacities of members and connection properties are assumed to be independent basic random variables, with normal distribution. The $\beta$-unzipping method, as described by Thoft-Christensen and Murotsu [6], is used to identify critical failure modes in the structural system. For each critical mode the probability of failure is calculated by using the method of reliability analysis of parallel systems. Then, by combination of the different failure modes as a series system and by using bounding techniques, the probability of failure of the overall structural system is estimated. In the optimization problem, the objective function is the weight of the structure, including the weight of the members and the weight of the connections, while the constraints are both deterministic and probabilistic.

\section{Structural analysis of semi-rigid steel frames}

The conventional analysis and design of steel frames are usually carried out under the assumption that the beam-column connections are either fully rigid or ideally pinned. However, the actual behavior of the beam-column connections in the steel frames is semi-rigid. The relationship between the beam end moment $(M)$ and the relative change in angle $\left(\theta_{r}\right)$ can be described by means of a typical moment-rotation curve. Some popular $M-\theta_{r}$ relations, such as linear, polynomial, exponential, and power models, are explained by Chen and Lui [7].

In the present research, the power model of Kishi et al. [4] and Chen and Lui [7] is used for describing the $M-\theta_{r}$ the relation of the connection with angles. The $M-\theta_{r}$ relation in this model is shown in eqn (1).

$$
\theta_{r}=\frac{M}{R_{k i}\left[1-\left(\frac{M}{M_{u}}\right)^{n}\right]^{1 / n}}
$$

In which $R_{k i}$ is the initial connection stiffness, $M_{u}$ is the ultimate moment capacity of the connection, and $\mathrm{n}$ is a shape parameter of the $M-\theta_{r}$ curve. The two parameters, $R_{k i}$ and $M_{u}$ are evaluated analytically based on the size and geometry of the connection, while the shape parameter $\mathrm{n}$ is obtained by curve- 
fitting technique. The effects of the connection flexibility are modeled by attaching rotational springs with stiffness modulus $R_{i}$ and $R_{j}$ at the ends $\mathrm{i}$ and $\mathrm{j}$ of a beam. For the sake of simplicity and for reducing the time of analysis, it is assumed that the rotational spring are linear with the stiffness modulus equal to $0.5 R_{k i}$ The stiffness matrix of a beam with rotational springs at the ends is function of the $R_{i}$ and $R_{j}$ as given by Hadianfard and Razani [5]. For columns, the stiffness matrix takes the usual form. The beams and the columns stiffness matrices can be assembled in the usual manner to form the stiffness matrix of the structure.

\section{Identification of critical failure modes}

The reliability of a structure is denoted by the factor $\mathfrak{R}$ which is defined by:

$$
\mathfrak{R}=1-P_{f}
$$

Where, $P_{f}$ is the probability that the structure will fail during the specified reference period. For a fundamental case (a structure with only two independent random variables $R$ and $S$, where $R$ is resistance variable and $S$ is load effect variable) the reliability index $\beta$ is defined by eqn (3).

$$
\beta=\frac{\mu_{M}}{\sigma_{M}}
$$

where $M=R-S$ is called the safety margin and $\mu_{M}, \sigma_{M}$ are the mean value and standard deviation of $M$. The probability of failure $\left(P_{f}\right)$ for this fundamental case can be calculated from eqn (4).

$$
P_{f}=\Phi(-\beta) \Leftrightarrow \beta=-\Phi^{-1}\left(P_{f}\right)
$$

where $\Phi$ is standard normal distribution function.

The $\beta$-unzipping method is one of the methods by which the reliability of the structures with ductile or brittle elements can be estimated at a number of different levels. The systems reliability at mechanism level is defined as the reliability of a series system with the significant mechanisms as elements. For generating the Fundamental mechanisms, the structure must be modeled by $\mathrm{n}$ failure elements. At first it is assumed that, the failure is accrued in critical failure element $l$ with the lowest reliability index, and structure is modified by removing the corresponding failure elements and adding a pair of so-called fictitious loads $\left(F_{l}\right)$ (normal forces or moments for ductile elements). The modified structure is then reanalyzed and new reliability indices are calculated for the others failure elements. If the $\beta$ for failure element $m$ was the lowest $\beta$ values. Then the failure is assumed in the elements $l$ and $m$ and the structure is modified and reanalyzed. In this way new $\beta$-values are calculated for all failure elements except $l$ and $m$ and the smallest $\beta$-value is calculated (for example in failure element $r$ ). In this way, 3 failure elements $l, m$ and $r$ are selected as part of failure tree. The same way can be used for selecting other failure elements of the failure tree. Formation of a mechanism can be unveiled by the fact that the 
corresponding stiffness matrix is singular. By this procedure a number of mechanisms with different numbers of failure elements will be identified. In this way, several re-analyses of the structure are necessary.

\section{Estimation of the probability of failure}

The reliability of a structural system can be estimated on the basis of a modeling by a series system where the elements in the series system are parallel systems (failure modes). Probability of failure for each parallel system $\left(P_{f p}\right)$ can be calculated by Ditlevsen's bounds. In this bounding method the lower and upper bounds are as following:

$$
\begin{gathered}
P_{f p(u)}=1-P\left[S_{1}\right]-\sum_{i=2}^{p} \operatorname{Max}\left\{0, \sum_{j=1}^{i-1} P\left[\bar{S}_{j} \cap S_{i}\right]-(i-2) P\left[S_{i}\right]\right\} \\
P_{f p(l)}=1-P\left[S_{1}\right]-\sum_{i=2}^{p} \operatorname{Min}\left\{P\left[\bar{S}_{j} \cap S_{i}\right]\right\}
\end{gathered}
$$

where, $P_{f p(u)}$ is upper bound and $P_{f p(l)}$ is lower bound of the probability of failure, $M_{i}$ is safety margin for failure element $i, P\left[S_{i}\right]$ is probability of occurrence of non-failure state in the failure element $i,\left(P\left(M_{i} \succ 0\right)\right)$ and $P\left[\bar{S}_{i}\right]$ is probability of occurrence of failure state in the failure element $i,\left(P\left(M_{i} \leq 0\right)\right)$ and $p$ is total number of failure elements. In these equations the $P\left[\bar{S}_{i} \cap S_{j}\right]$ can be calculated as below:

$$
P\left[\bar{S}_{i} \cap S_{j}\right]=P\left[\left(M_{i} \leq 0\right) \cap\left(M_{j}>0\right)\right]=1-P\left[S_{i}\right]-P\left[\bar{S}_{i} \cap \bar{S}_{j}\right]
$$

where $P\left[\bar{S}_{i} \cap \bar{S}_{j}\right]$ is joint probability and it can be estimated as following:

$$
\begin{gathered}
\operatorname{Max}\left(P_{1}, P_{2}\right) \leq P_{i j}=P\left[\bar{S}_{i} \cap \bar{S}_{j}\right] \leq P_{1}+P_{2} \\
P_{1}=\Phi\left(-\beta_{i}\right) \Phi\left(-\gamma_{j}\right), P_{2}=\Phi\left(-\beta_{j}\right) \Phi\left(-\gamma_{i}\right) \\
\gamma_{i}=\frac{\beta_{i}-\rho_{i j} \beta_{j}}{\sqrt{1-\rho_{i j}^{2}}}, \gamma_{j}=\frac{\beta_{j}-\rho_{i j} \beta_{i}}{\sqrt{1-\rho_{i j}^{2}}}
\end{gathered}
$$

where $\rho_{i j}$ is correlation coefficient as defined by eqn (11).

$$
\rho_{i j}=\frac{\operatorname{Cov}\left[M_{i}, M_{j}\right]}{\sigma_{M i} \sigma_{M j}} \quad i \neq j
$$

For calculation of probability of failure of the structural system (series system $\left.\left(P_{f s}\right)\right)$ the simple bounds method (Cornell's bounds) as defined by eqn (12) can be used.

$$
\operatorname{Max}_{i=1}^{n}\left(P_{i}\right) \leq P_{f s} \leq 1-\prod_{i=1}^{n}\left(1-P_{i}\right)
$$

where $P i$ is probability of failure of the element $\mathrm{i},\left(P\left(M_{i} \leq 0\right)\right)$. 


\section{Formulation of RBDO problems}

The optimal design problem can be formulated mathematically as:

$$
\begin{cases}\text { Minimize } & W\left(X_{1}, X_{2}, \ldots, X_{n}\right) \\ \text { Such that } & P_{f s}\left(X_{1}, X_{2}, \ldots, X_{n}\right) \leq P_{f a}\end{cases}
$$

Where, $\mathrm{W}$ is weight of the structural system consist of weight of the structural members $(W s)$ and weight of the connections $(W c)$. The parameter $X i$ is design variable, $P_{f s}$ is probability of failure of the structural system and $P_{f a}$ is a specified allowable probability of failure.

The structural weight can be obtained from eqn (14).

$$
W=W_{S}+W_{C}=\sum_{i=1}^{n} \rho_{i} L_{i} A_{i}+\sum_{i=1}^{m} \rho_{i} V_{C i}
$$

where $\rho_{i}, l_{i}, A_{i}$ are the density, length and the cross-sectional area of the member $\mathrm{i}$, and $V_{C i}$ is the volume of the connection $\mathrm{i}$, and $n, m$ are the total number of members and connections respectively. In a structural system with constant density for all members and connections, the objective function can be shown as:

$$
\text { Minimize } \quad V\left(A_{i}, K_{i}, M_{u i}\right)=\sum_{i=1}^{n} L_{i} A_{i}+\sum_{i=1}^{m} V_{C i}\left(K_{i}, M_{u i}\right)
$$

Then the design variables are: $A_{i}, K_{i}, M_{u i}$ where, $K i$ and $M u i$ are the beamcolumn connection properties (stiffness and moment capacity).

The volume of the connections $\left(V_{C i}\right)$ is function of the type, shape, flexibility, capacity and the others properties of the connections. The type and the shape of the connections must be selected before the optimization process, and the others properties can be obtained from the $M-\theta_{r}$ relation of the connection. For reducing the number of the design variables, the moment capacity of the connection can be considered as a function of connection stiffness $(K i)$. Then the design variables are only $A i$ and $K i$, and these variables can be obtained from solving the RBDO problem.

\section{Solving the optimization problem}

The constrained nonlinear optimization problem of eqn (13) can be changed in to an unconstrained optimization problem using method of interior penalty function [1]. In the penalty function method, a penalty term that takes care of the constraints is added to the original objective function.

Consider the original optimization problem as eqn (16).

$$
\begin{cases}\text { Minimize } & f(X) \\ \text { Such that } & g_{j}(X) \leq 0 \quad j=1,2, \ldots, m\end{cases}
$$

where $f(x)$ is objective function and $g_{j}(X),(j=1,2, \ldots, m)$ are constraints of the optimization problem. 
Thus, the transformed problem is as following:

$$
\text { Minimize } \phi\left(X, r_{k}\right)=f(X)-r_{k} \sum_{j=1}^{m} \frac{1}{g_{j}(X)} \quad k=1,2,3, \ldots
$$

where $r_{k}$ is the penalty parameter.

The solution process is as follows: Initial value of $\{X\}$ and $r$ are chosen. $\{X\}$ must lie in the feasible region and $r$ must be a positive number. For the given $r_{k}$, the objective function $\phi\left(X, r_{k}\right)$ is minimized to obtain $\left\{X^{*}\right\}$ and the convergence criterion of $\left\{X^{*}\right\}$ to the optimum is checked. If it is not satisfied, the parameter $r$ is reduced $\left(r_{k+1}=c r_{k}, c<1\right)$ and starting from $\left\{X^{*}\right\}$ the function $\phi\left(X^{*}, r_{k+1}\right)$ is minimized again. The step of reducing $r$ and minimizing $\phi\left(X, r_{k}\right)$ are repeated until the convergence criterion is satisfied.

In each step of the penalty function technique, the obtained unconstrained optimization problem can be solved by using a direct search method as Powell or Rosenbrock method. For example the solution process for the Powell's method is as follows: 1-Choose an initial vector $\{X\}$ and $n$ initial independent directions $\{S q\}, q=1,2, \ldots, n$. 2- Select new vector $\{Y\}$ equal to $\{X\}$. 3- Find $\alpha^{*}$ to minimize $F(\{X\}+\alpha\{S q\})$ and set $\{X\} \leftarrow\{X\}+\alpha^{*}\left\{S_{q}\right\}$ for $q=1,2, \ldots, n$. 4-Set $\left\{S_{n+1}\right\} \leftarrow\{X\}-\{Y\}$

and find $\alpha^{*}$ to minimize $F\left(\{X\}+\alpha\left\{S_{n+1}\right\}\right)$, and set $\{X\} \leftarrow\{X\}+\alpha^{*}\left\{S_{n+1}\right\}$. 5- Replace $\left\{S_{q}\right\} \leftarrow\left\{S_{q+1}\right\}$ for $q=1,2, \ldots, n$. 6- Repeat from step 2 .

\section{Numerical calculations and discussion}

In this section some examples of numerical calculations are presented. These examples show applicability of the proposed methodology and importance of the consideration of semi-rigid behavior of the connections in the RBDO problems.

Example 1: A steel frame, as shown in figure 1, is assumed to be under vertical and lateral loads. External loads are random variables with mean values as given in figure 1. Also member resistances and moment capacities of the connections are random variables. All of the random variables are normally distributed with coefficient of variation equal to 0.1 . Mpa.

It is assumed that the expected value for yield strength of the steel is $\sigma y=240$

In this example, nine failure elements are considered in the reliability analysis. Four failure elements are in the beam, namely a yield hinge possibility at each end of the beam and another yield hinge at middle of the beam and the possibility of failure in axial force. Also each column has two failure elements, namely a yield hinge possibility at the end of the column (near the beam) and the possibility of failure in axial force. Another failure element is possibility of failure in axial force in the bracing element.

The RBDO problem can be formulated as following:

$$
\text { Minimize } V\left(A_{i}, K_{i}\right)=V_{S}\left(A_{i}\right)+V_{C}\left(K_{i}\right)=\sum_{i=1}^{n} L_{i} A_{i}+\sum_{j=1}^{m} V_{C j}\left(K_{j}\right)
$$


Such that:
1) $\frac{P_{f s}}{P_{f a}}-1 \leq 0$,
2) $1-\frac{A_{i}}{A_{i, \min }} \leq 0 \quad, i=1,2, \ldots, n$
3) $1-\frac{K_{j}}{K_{j, \min }} \leq 0 \quad, j=1,2, \ldots, m$,
4) $\frac{K_{j}}{K_{j, \max }}-1 \leq 0, j=1,2, \ldots, m$

where $A i, L i$ are area and length of the member $i, K j$ is rotational stiffness of the connection $j\left(K_{j}=0.5 R_{k i}\right), A i$ min is minimum cross sectional area of the member $i$ (it is selected by designer before optimization). The parameters $K_{j, m i n}, K_{j, \max }$ are minimum and maximum rotational stiffness of the connection $j$. These values can be selected with respect to the shape and type of the connections by using appropriate $M-\theta_{r}$ relation. By selecting the cross-sectional area and the shape of the section (for example: IPE, IPB, UNP and etc.) the others geometrical properties of the section, such as: moment of inertia, plastic modulus and etc. can be evaluated.

It is assumed that the connection weight (volume) is function of the connection stiffness. Then for fully pinned connection, the weight of the connection is minimum and for fully rigid connection, it is maximum. Relation between the connection volume $\left(V_{c j}\right)$ and the connection stiffness $\left(K_{j}\right)$ can be written as below:

$$
V_{C j}=\alpha_{j}\left[V_{j, \text { min }}+C_{j}\left(K_{j}-K_{j, \text { min }}\right)\right] \leq V_{j, \text { max }}, \quad C_{j}=\frac{V_{j, \text { max }}-V_{j, \text { min }}}{K_{j, \text { max }}-K_{j, \text { min }}}
$$

where $V_{j, \min }, V_{j, \max }$ are the minimum and maximum volume of each connection, and $\alpha$ is constructional factor $(\alpha \geq 1)$. This factor can be used for consideration the difficulties in the construction of the connections.

Also relation between the moment capacity $(M u)$ and the stiffness $(K)$ of the connection can be written as:

$$
M_{u}=M_{u, \min }+\frac{M_{u, \text { max }}-M_{u, \text { min }}}{K_{\text {max }}-K_{\text {min }}}\left(K-K_{\text {min }}\right)
$$

where $M_{u, \min }, M_{u, \max }$ are minimum and maximum moment capacities of the connections (it is selected by designer).

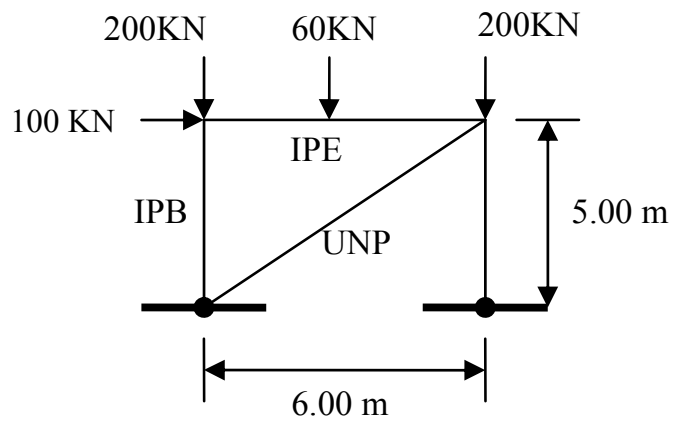

Figure 1: $\quad$ Steel frame of the example 1. 
Some different types of the beam-column connections with angles are considered in this example and for each case the RBDO problem is solved.

a) Frame with fully pinned connections: In this ideal case, the initial stiffness and the moment capacity of the connections are zero and connection weight (volume) is at least. The following limitations are considered in this example.

$$
\begin{aligned}
& A_{c, \text { min }}=26 \mathrm{~cm}^{2}, \quad A_{b, \text { min }}=10 \mathrm{~cm}^{2}, \quad A_{b r, \text { min }}=12 \mathrm{~cm}^{2} \\
& K_{\text {min }}=K_{\text {max }}=0, \quad M_{u, \text { min }}=M_{u, \text { max }}=0 \\
& V_{c, \text { min }}=100 \mathrm{~cm}^{3}, \quad V_{c, \text { max }}=200 \mathrm{~cm}^{3}, \quad \alpha=1.20, \quad P_{f a}=0.001
\end{aligned}
$$

where $A c, A b, A b r$ are cross-sectional area of the columns, beam and bracing. Initial values for the design variables are selected as: $A_{c}=50 \mathrm{~cm}^{2}, A_{b}=90 \mathrm{~cm}^{2}, A_{b r}=13 \mathrm{~cm}^{2}$

The optimum solution can be found by solving the RDBO problem, and the results are as: $A_{c}^{*}=26 \mathrm{~cm}^{2}, A_{b}^{*}=52.2 \mathrm{~cm}^{2}, A_{b r}^{*}=12 \mathrm{~cm}^{2}$.

In this case the minimum volume of the structure without consideration the connection volume is $V_{\min , 1}=66692 \mathrm{~cm}^{3}$, and with consideration the volume of the connections is $V_{\min , 2}=67052 \mathrm{~cm}^{3}$.

b) Frame with single web angle connections: In conventional ways this type of the connection was assumed to behave as a fully pinned connection, but the real behavior of the connection is semi-rigid and this behavior can be shown by the Kishi and Chen power model (eqn (1)).

For example in a web connection by angle $L 100 \times 100 \times 10 \mathrm{~mm}$ with length $l=12 \mathrm{~cm}$ the parameters of Kishi and Chen model are as:

$R_{k i}=1.92 \times 10^{3} \mathrm{KN} . \mathrm{m} / \mathrm{rad}, M_{u}=4.6 \mathrm{KN} . \mathrm{m}$. In this case the volume of each connection is about $V_{c i}=230 \mathrm{~cm}^{3}$. The upper and lower bounds for these parameters can be obtained by changing the size and the length of the connection angle. Therefore the following limitations may be assumed for the connections.

$$
K_{\text {min }}=0.25 \times 10^{3}, K_{\text {max }}=3.5 \times 10^{3}, M_{u, \text { min }}=2, M_{u, \text { max }}=14, V_{c, \text { min }}=150, \quad V_{c, \text { max }}=400, \quad \alpha=1.20
$$

By selecting the suitable initial values as: $A_{c}=40, A_{b}=60, A_{b r}=20, K=1 \times 10^{3}$ the optimum solution can be found as:

$$
A_{c}^{*}=26 \mathrm{~cm}^{2}, A_{b}^{*}=48.6 \mathrm{~cm}^{2}, A_{b r}^{*}=12 \mathrm{~cm}^{2}, K^{*}=1.31 \times 10^{3}, V_{\min , 1}=64532, V_{\min , 2}=65012
$$

The optimum volume in this manner is less than the optimum volume of the case (a).

c) Frame with double web angles connections: In this type of the connection the limits on the connection properties are two times the values of the case (b). The optimum solution is given in table 1 .

d) Frame with top and seat angles connections: The properties of these semi-rigid connections can be found by Kishi and Chen model. For example, in a connection by the angles $L 100 \times 100 \times 10 \mathrm{~mm}$ with length $l=12 \mathrm{~cm}$ and with beam depth equal to $24 \mathrm{~cm}$, the initial stiffness is $R_{k i}=9.79 \times 10^{3} \mathrm{KN} . \mathrm{m} / \mathrm{rad}$ and the moment capacity is $M_{u}=27.4 \mathrm{KN} . \mathrm{m}$ and the volume of each connection is 
about $V_{c i}=460 \mathrm{~cm}^{3}$. Then the constraints on the connection properties may be selected as:

$$
K_{\text {min }}=3.0 \times 10^{3}, K_{\max }=9.0 \times 10^{3}, M_{u, \text { min }}=15, M_{u, \text { max }}=45, V_{c, \text { min }}=300, V_{c, \text { max }}=800
$$

The optimum solution of this case is given in table 1.

Table 1: $\quad$ Results of the RBDO for the frame of example 1.

\begin{tabular}{|l|l|l|l|l|l|l|}
\hline Connection type & $\begin{array}{l}A_{c}^{*} \\
\left(\mathrm{~cm}^{2}\right)\end{array}$ & $\begin{array}{l}A_{b}^{*} \\
\left(\mathrm{~cm}^{2}\right)\end{array}$ & $\begin{array}{l}A_{b r}^{*} \\
\left(\mathrm{~cm}^{2}\right)\end{array}$ & $\begin{array}{l}V_{\min , 1} \\
\left(\mathrm{~cm}^{3}\right)\end{array}$ & $\begin{array}{l}V_{\min , 2} \\
\left(\mathrm{~cm}^{3}\right)\end{array}$ & $\begin{array}{l}K_{\text {opt }} \\
(\mathrm{KN} . \mathrm{m} / \mathrm{rad})\end{array}$ \\
\hline a) fully pinned & 26 & $\begin{array}{l}52 . \\
5\end{array}$ & 12 & 66692 & 67052 & 0 \\
\hline b) single web angle & 26 & $\begin{array}{l}48 . \\
6\end{array}$ & 12 & 64532 & 65012 & 1000 \\
\hline c) double web angle & 26 & $\begin{array}{l}46 . \\
7\end{array}$ & 12 & 63429 & 64389 & 2000 \\
\hline d) seat angle & 26 & $\begin{array}{l}46 . \\
5\end{array}$ & 12 & 63273 & 64392 & 5000 \\
\hline $\begin{array}{l}\text { e) seat angle }+ \text { web } \\
\text { angles }\end{array}$ & 26 & $\begin{array}{l}46 . \\
5\end{array}$ & 12 & 63273 & 65937 & 7000 \\
\hline f) fully rigid & 26 & $\begin{array}{l}46 . \\
5\end{array}$ & 12 & 63273 & 71790 & $\infty$ \\
\hline
\end{tabular}

e) Frame with top and seat angles with double web angles connections:

For a connection by angles $L 100 \times 100 \times 10 \mathrm{~mm}$ with length $l=12 \mathrm{~cm}$ and with beam depth equal to $24 \mathrm{~cm}$, the initial stiffness is $R_{k i}=14.6 \times 10^{3} \mathrm{KN} . \mathrm{m} / \mathrm{rad}$ and the moment capacity is $M_{u}=48.5 K N . m$. Then the constraints on the connection properties may be selected as:

$$
K_{\text {min }}=5.0 \times 10^{3}, K_{\text {max }}=12 \times 10^{3}, M_{u, \text { min }}=30, M_{u, \text { max }}=70, V_{c, \text { min }}=600, V_{c, \text { max }}=1500
$$

The optimum solution of this case is given in table 1 .

f) Frame with fully rigid connections: In this ideal case, it is assumed that the initial stiffness of the connections is infinite (a large number as $K=1 \times 10^{6} \mathrm{KN} . \mathrm{m} / \mathrm{rad}$ ) and the moment capacity of the connections is greater than the moment capacity of the connected beam. Also in this case the connection weight (volume) is maximum and it is about $V_{c i}=1800 \mathrm{~cm}^{3}$. Because of difficulty in the construction of the rigid connections, the parameter $\alpha$ is assumed to be $\alpha=1.30$. The results of the RBDO are shown in the table 1 . It can be seen from the table 1 that by increasing the connection stiffness, the weight (volume) of the structural members will be decreased but the weight of the connections will be increased. Then at the optimum connection stiffness, the total weight of the structural system (consist weight of the connections) will be at least. The case c (connection by double web angles) is an optimum manner between all of the cases. Also this table appears that the weight of the overall structure in the cases of fully pinned and fully rigid is greater than the weight of 
the other cases. Therefore, ignoring the weight and the behavior of the semi-rigid connections can lead to the unrealistic optimum solution in the steel frames.

Example 2: A steel frame as shown in figure 2 is assumed to be under uniform vertical loads with expected value $15 \mathrm{KN} / \mathrm{m}$. It is assumed that the coefficient of variation for all of the random variables is equal to 0.1 . All beamcolumn connections are similar and made of angles. Basic design variables are rotational stiffness of the connections $(\mathrm{K})$ and cross-sectional area of the members as: Ac1: corner columns in the first story, Ac2: middle column in the first story, Ac3: corner columns in the second story, Ac4: middle column in the second story, $\mathrm{Ab} 1$ : beams in the first story, $\mathrm{Ab} 2$ : beams in the second story.

The constraints of this example are: $A_{c, \text { min }}=26 \mathrm{~cm}^{2}, A_{b, \text { min }}=10 \mathrm{~cm}^{2}, P_{f a}=0.001$.

The results of the RBDO for different type of the connections are shown in table2. This table indicates that the case (d) is an optimum case, and the weight (volume) of the overall structure in this case is at least.

Table 2: $\quad$ Results of the RBDO for the frame of example 2.

\begin{tabular}{|c|c|c|c|c|c|c|c|c|c|}
\hline $\begin{array}{l}\text { Connect } \\
\text { ion } \\
\text { type }\end{array}$ & $\begin{array}{l}A_{c 1}^{*} \\
\left(\mathrm{~cm}^{2}\right)\end{array}$ & $\begin{array}{l}A_{c 2}^{*} \\
\left(\mathrm{~cm}^{2}\right)\end{array}$ & $\begin{array}{l}A_{c 3}^{*} \\
\left(\mathrm{~cm}^{2}\right)\end{array}$ & $\begin{array}{l}A_{c 4}^{*} \\
\left(\mathrm{~cm}^{2}\right)\end{array}$ & $\begin{array}{l}A_{b 1}^{*} \\
\left(\mathrm{~cm}^{2}\right)\end{array}$ & $\begin{array}{l}A_{b 2}^{*} \\
\left(\mathrm{~cm}^{2}\right)\end{array}$ & $\begin{array}{l}V_{\min 1} \\
\left(\mathrm{~cm}^{3}\right)\end{array}$ & $\begin{array}{l}V_{\min , 2} \\
\left(\mathrm{~cm}^{3}\right)\end{array}$ & $\begin{array}{l}K_{\text {opt }} \\
(\mathrm{KN} \cdot \mathrm{m} / \mathrm{rad})\end{array}$ \\
\hline (a) & 26 & 26 & 26 & 26 & 31 & 31 & $\begin{array}{l}11040 \\
0\end{array}$ & $\begin{array}{l}11184 \\
0\end{array}$ & 0 \\
\hline (b) & 26 & 26 & 26 & 26 & 27 & 27 & $\begin{array}{l}10368 \\
0\end{array}$ & $\begin{array}{l}10560 \\
0\end{array}$ & 1000 \\
\hline (c) & 26 & 26 & 26 & 26 & $\begin{array}{l}25 . \\
5\end{array}$ & $\begin{array}{l}25 . \\
5\end{array}$ & $\begin{array}{l}10146 \\
0\end{array}$ & $\begin{array}{l}10530 \\
0\end{array}$ & 2000 \\
\hline (d) & 26 & 26 & 26 & 26 & $\begin{array}{l}21 . \\
5\end{array}$ & $\begin{array}{l}22 . \\
5\end{array}$ & 94238 & 98712 & 5000 \\
\hline (e) & 26 & 26 & 26 & 26 & 19 & 21 & 90485 & 99912 & 7000 \\
\hline (f) & 26 & 26 & 26 & 26 & $\begin{array}{l}16 . \\
5\end{array}$ & $\begin{array}{l}18 . \\
5\end{array}$ & 86100 & $\begin{array}{l}10482 \\
0\end{array}$ & $\infty$ \\
\hline
\end{tabular}

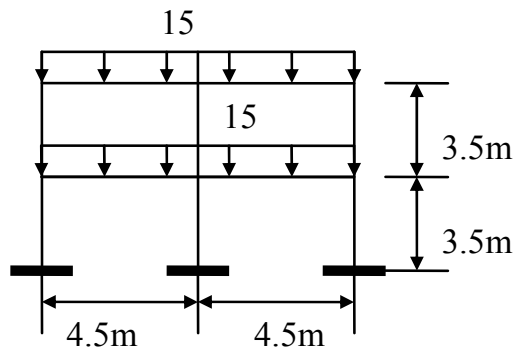

Figure 2: $\quad$ Steel frame of example 2.

Example 3: In this example a two-story steel frame is considered as shown in figure 3. The mean values of the loads variables are as: $P_{1}=169 \mathrm{KN}, P_{2}=89 \mathrm{KN}$, $P_{3}=116 \mathrm{KN}, P_{4}=31 \mathrm{KN}$. The coefficient of variation for $P_{2}, P_{3}$ and $P_{4}$ is equal 
to 0.25 and for the others loads and design variables is equal to 0.15 . As shown in the figure 3 the number of potential yield hinge is $n=19$. In this example six design variables are considered. One is rotational stiffness of the connections $(\mathrm{K})$ and the others are cross-sectional area of the members as: Ac1: columns in the first story, Ac2: columns in the second story, Ab1: left beam in the first story, Ab2: right beam in the first story, Ab3: beam in the second story.

All connections are end plates with moment capacity equal to the plastic moment of the connected beam. This type of the connection is usually assumed to be fully rigid. However, the actual behavior of this connection is semi-rigid and can be modeled by Fry and Morris polynomial model as described by Chen and Lui [7]. For the end plate connection with thickness $t p=2.5 \mathrm{~cm}$ and height $\mathrm{dg}=50 \mathrm{~cm}$, the initial stiffness of the connection is equal to $R_{k i}=8.2 \times 10^{4} \mathrm{KN} . \mathrm{m} / \mathrm{rad}$. Two types of the connection behavior are considered. One is fully-rigid (ideal behavior) and another is semi-rigid behavior (actual behavior). Many different allowable probability of failure are considered. Results of the optimization process are presented in table 3 for some values of the $P_{f a}$. Also the variation of the optimum volume is presented with respect to the $P_{f a}$ in figure 4. Results show that, the optimum weight (volume) of the overall structure in the case of fully-rigid is less than the case of semi-rigid. Then the assumption of fully rigid connections in the RBDO is unsafe and may lead to unrealistic results.

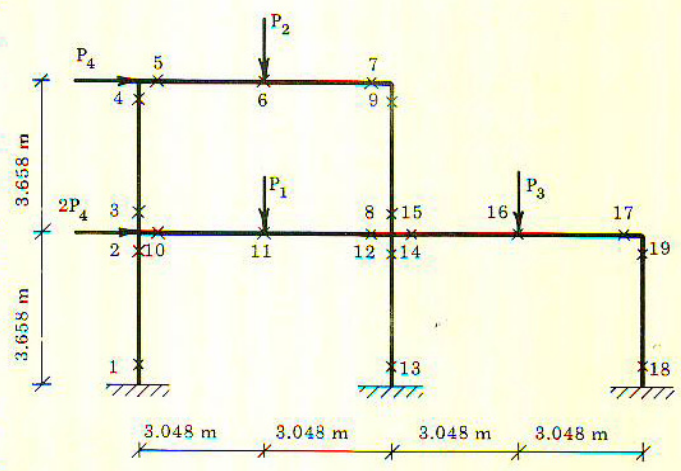

Figure 3: $\quad$ Steel frame of example 3.

Table 3: $\quad$ Results of the RBDO for the frame of example 3.

\begin{tabular}{|c|c|c|c|c|c|c|c|c|c|c|c|c|}
\hline \multirow{2}{*}{$\begin{array}{l}\text { Connec- } \\
\text { tion } \\
\text { Type }\end{array}$} & \multicolumn{6}{|c|}{$P_{f a}=0.001$} & \multicolumn{6}{|c|}{$P_{f a}=0.0001$} \\
\hline & $\mathrm{C} 1$ & $\mathrm{C} 2$ & B1 & B2 & B3 & $V \min$ & $\mathrm{C} 1$ & $\mathrm{C} 2$ & B1 & B2 & B3 & Vmin \\
\hline $\begin{array}{l}\text { Fully- } \\
\text { rigid }\end{array}$ & 601.1 & 64.4 & 70.6 & 74.8 & 72.7 & 267473 & 82.7 & 80.1 & 79.4 & 78.4 & 80.6 & 316135 \\
\hline $\begin{array}{l}\text { Semi- } \\
\text { rigid }\end{array}$ & 70.1 & 55.3 & 81.6 & 76.9 & 73.1 & 280019 & 83.5 & 82.2 & 89.4 & 88.5 & 85.3 & 333667 \\
\hline
\end{tabular}




\section{Conclusions}

In this research, the realistic semi-rigid behavior of the beam-column connections is considered in the RBDO problem. The total structural weight (consist of the connections weight) is minimized while the probability of failure of the structure did not exceed a prescribed acceptable value. The numerical examples indicate the applicability of the proposed method. Also theses examples indicate the importance of the assumption of semi-rigid behavior of the connections in the RBDO. The assumption of fully-pinned connections is usually safe and over design but the assumption of fully-rigid connections is usually unsafe and under design. Further by the proposed method; the optimum stiffness of the connections can be evaluated. This optimum connection stiffness can lead to minimum weight of the structure.

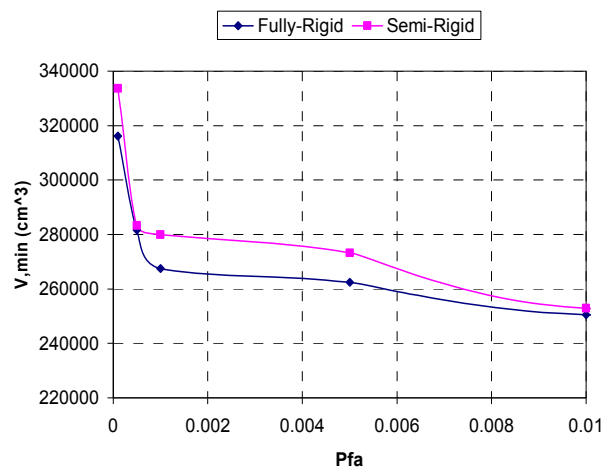

Figure 4: Optimum volume of the frame of example 3 for the different $P_{f a}$.

\section{References}

[1] Kirsch, U., Optimum Structural Design, McGraw-Hill Book Company, 1981.

[2] Haftka, R.T., Gurdal, Z., Elements of Structural Optimization, Kluwer Academic Publishers, Netherlands, 1992.

[3] Nakib, R., Frangopol, D., Reliability-based structural optimization using interactive graphics, Computers \& Structures, Vol. 37, pp. 27-34, 1990.

[4] Kishi, N., Chen, W.F., Goto, Y. \& Matsuoka, K.G., Design aid of semi-rigid Connections for frame analysis, Engineering Journal, AISC, Vol. 30, No. 3, pp. 90-107, 1993.

[5] Hadianfard, M.A., Razani, R., Effects of semi-rigid behavior of connections in the reliability of steel frames, Structural Safety, 25, pp.123-138, 2003.

[6] Thoft-Christensen, P., Murotsu, Y., Application of Structural System Reliability theory, Springer-Verlag, Berlin, Heidelberg, 1986.

[7] Chen, W.F., Lui, E.M., Stability Design of Steel Frames, CRC Press, Inc., 1991. 